\title{
Building Blocks for Eco-efficient Cloud Computing for Higher Learning Institutions in Tanzania
}

\author{
Rodrick Frank Mero \\ Department Information Communication Science and Engineering \\ NM-AIST \\ Arusha , Tanzania
}

\begin{abstract}
Owning and managing a cloud-computing infrastructure, i.e. private data centers (DC), is a feasible way forward for an organization to ensure security of data when opting for cloud computing services. However, the cost associated with operating and managing a DC is a challenge because of the huge amount of power consumed and the carbon dioxide added to the environment. In particular, Higher Learning Institutions in Tanzania (HLIT) are among the institutions which need efficient computing infrastructure. This paper proposes ecoefficient cloud computing building blocks that ensure environment protection and optimal operational costs of a cloud computing framework that suffices HLIT computing needs. The proposed building blocks are in a form of power usage (renewable and nonrenewable); cloud deployment model and data center location; ambient climatic conditions and data center cooling; network coverage; quality of service and HLIT cloud software. The blocks are identified by considering HLIT computing requirements and challenges that exist in managing and operating cloud data centers. Moreover, this work identifies the challenges associated with optimization of resource usage in the proposed approach; and suggests related solutions as future work.
\end{abstract}

Keywords:Cloud computing, Building Blocks, Higher Learning Institution in Tanzania, Power consumption, eco-efficient, Traditional computing, Data Center

\section{INTRODUCTION}

Cloud computing has been trusted as a technology of choice in most resource provisioned in academic environments $[1,2,3]$. Despite the flexibility and cost effective services offered by cloud computing, none of the Higher Learning Institutions in Tanzania (HLIT) has jumped onto the bandwagon. These institutions still use traditional computing (TC) that has been proven to be uneconomical in terms of maintenance and software purchase costs [4]. Moreover, the shortage of stable power supply prevalent in the country and lack of ample funding faced by most HLIT necessitate the development of computing solutions which are eco-efficient.

In order to develop an eco-efficient cloud computing framework that suits an academic environment, there is a need for addressing existing computing challenges $[5,6]$. These include high operational costs and carbon dioxide emissions by cloud data centers, and poor guarantee in quality of service (QoS) as per Service Level Agreements (SLA). Therefore, the building blocks of the framework should be able to address these challenges.

In general, building blocks are all requirements such as: technology, policies, cloud best practices, software and infrastructure that support in building and adoption of economical, efficient and environment friendly cloud computing solutions. Normally, cloud architecture is made of layers; each layer is designed to provide a service such as Infrastructure-as-aService (IaaS), Platform-as-a-Service (PaaS) and Software-as-aService (SaaS) [7]. Thus, eco-efficient building blocks should also be able to address the complexity of each layer according to the underlying cloud computing architecture.

\section{Principles for eco-efficient building block selection.}

Design principles that guide selection of specific ecoefficient building blocks are characterized by features which consider the economical, technological and environmental challenges that exist in the designated environment for cloud computing services. The criteria for eco-efficient building blocks selection are as follows:

a) HLIT as other academic institutions in the world are in need of cost effective solution to their existing computing challenges, therefore, focusing on free and open source software (FOSS) is an important criterion in selecting cloud software. Likewise, choosing cloud deployment model should consider its operation costs and challenges relative to a Traditional Computing (TC) system.

b) Huge power consumption and environmental pollution by current cloud data centers have been a challenge to data center operators; thus focusing on green aware and low power consumption technologies is an important criterion.

c) Efficiency and flexibility of computing solution are very important for its acceptance; thus cloud computing building blocks should guarantee quality of service. Accordingly, network connections must ensure reliability and availability of cloud computing services to end users. Moreover, virtual machine consolidation should not impact performance of cloud services.

To this end, this work has identified building blocks needed for developing eco-efficient cloud computing that aligns with the guidelines and issues related to green power utilization.

The remaining sections of this work are organized as follows: Section 2 presents eco-efficient building blocks that are necessary to align with eco-efficient cloud requirements for HLIT, Section 3 presents related works and Section 4 concludes and discusses the work while suggesting future directions.

\section{Eco-efficient Building Blocks}

As mentioned in previous section eco-efficient cloud computing building blocks refer to all requirements such as technology, policies, cloud best practices, software and infrastructure that support building and adoption of economical, efficient and 
environment friendly cloud computing. In this chapter, we focus on environmental, technological and infrastructure building blocks.

Generally, cloud computing is made up of data centers that are connected using high speed network connections [8]. Each data center is made up of a large number of hosts, in each host there are virtual machines (VMs) that act as user PCs. VMs run as normal processes in computing systems. What distinguishes VMs from normal process is their capability to virtually work like physical hardware with operating system (OS), memory, processor, and storage. Thus, the act of purchasing a new PC in a TC scenario can be replaced by a request for a new virtual machine in the cloud.

To ensure efficient and green aware computing in HLIT, an eco-aware cloud framework is inevitable. Development of such a green cloud needs clear identification of well suited technological building blocks (i.e., networks, efficient cooling infrastructure, operating system (OS), cloud management tools) in all layers of the cloud architecture (i.e., IaaS, PaaS, SaaS). As academic ecoefficient cloud-computing services expect to be accessed by a large number of users, especially students and researchers, efficiency and scalability of cloud resources in such multiuser and dynamic environment have to be guaranteed.

For efficient cloud operation and coordination of data center resources, we identify and propose the building blocks that suffice HLIT cloud computing needs, see Fig 1. The building blocks are in the form of nature of power source (i.e., renewable and nonrenewable); deployment model and data center location; ambient climatic conditions and data center cooling; network coverage; quality of service and HLIT cloud software.

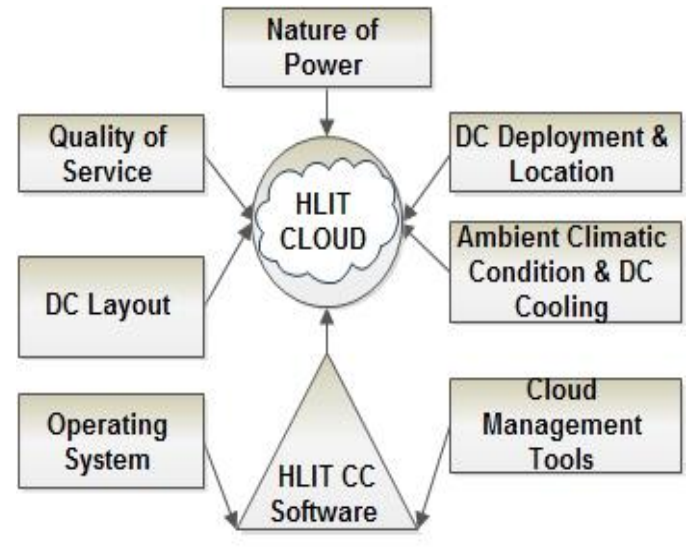

Fig 1 Building Blocks

\subsection{Form / nature of Power}

Tanzania like other developing countries has a low level of electrification [9] while the need for electric power consumption is increasing year after year $[10,11]$. As cloud data center power consumption could be similar to that needed to power a city, the need to consider energy efficiency becomes very important. Otherwise, providers would incur huge operational costs to maintain data centers (Markoff \& Lohr, 2009). Therefore, consideration of renewable energy sources becomes very important in avoiding a huge amount of carbon emission from bio-fuels.

Today's data centers are powered by different sources of energy. Data centers draw power from local electric grids. These grids are powered by either fossil-fuel based brown energy, renewable resource based on green energy or more commonly a combination of both. Hydro power plants are among few electric power production centers in Tanzania. Hydro production centers are located at Nyumba ya Mungu, Kidatu, and Mtera. Other sources of electrical power that are connected into the national grid are from fossil-fuel sources (petroleum, diesel); for example, power generated by Songas Company from Ubungo, Tegeta and other locations. It is difficult to distinguish power from renewable sources and fossil-fuel in the national grid [8] Although to locate a data center near Kidatu or Nyumba ya Mungu regions will result in more usage of renewable energy than placing them close to fossil fuel power sources [12]. Similarly, processing user request by considering closest path can bring challenges because a large number of users' requests can overload a single data center. Comparing data-centers located closer to renewable power production sites (i.e. Kidatu, Mtera) with ones located close to nonrenewable power production sites (i.e. Tegeta and Ubungo); access frequency of virtual machines located in Ubungo is considered to be higher due to expected large number of requests from HLIT closer to that area.

Therefore, challenges are in terms of optimization of workload, power consumption and carbon dioxide emission. Moreover, temperature and drought seasons bring challenges to hydro power generation.

The proposed approach in this chapter is to utilize both renewable and nonrenewable power sources appropriately. In the same vein, developing a Heuristic that can minimize tradeoff between power consumption, quality of service and carbon emission in such environment is an important concern. For example, migrating VMs from over-provisioned hosts in Ubungo data center to under provisioned data center in Kidatu or Nyumbaya Mungu should result into reduction of considerable amount of carbon emission; but leads to tradeoff between green aware consolidation and migration costs.

\subsection{Cloud Deployment model and data center location}

As discussed in [4], the Hybrid model is the preferred deployment model for HLIT. This model is suitable for academic environment because it combines advantages that exist in public and private cloud [13]. Hybrid model maintains private data center that can support complex mixed workloads. This model makes utilization of local IT infrastructure as private cloud with other selected service from public. As observed in [4], HLIs are facing challenges such as lack of constant power supply, inefficient cooling systems, and redundant of inefficient IT infrastructure. These issues raise concerns on capability for an institution to own and manage a private data center. The cost effective and reliable way is to locate a private data-center in a zone where it can be accessed by HLIs with optimal access costs. This strategy minimizes data center operational costs that could be incurred by a single institution.

Geographical location of a data center plays an important role. A data center located in an area with easy access to hydro power, for example, would have a lower carbon footprint than a data center located in an area that depends on coal, oil, or natural gas [14]. In the HLIT hybrid cloud model, private data center should be positioned by considering factors such as availability of renewable energy resources, availability of enough bandwidth, i.e. accessibility to the National ICT Broadband Backbone (NICTBB). It is important to locate a data center in a region where temperature is very low in order to reduce the need of having large 
number of cooling systems that can increase data center energy consumption. A geographical location that experiences high temperature and humidity levels will consume more energy as the data center physical infrastructure systems work harder to maintain consistent, moderate temperature and humidity levels [14]. Seemingly, locating data center close to renewable resources will make utilization of energy/ power from green energy sources that results in reduction of carbon footprint into the environment. From this point of view, data center location, IT load, and electrical efficiency are important factors to be considered when building and managing a data center.

\subsection{Ambient climatic conditions and data center cooling.}

The Green Grid [15] outlined seven strategies to improve data center efficiency. One of the proposed practices which can lead to improved power consumption is the use of free cooling. The American Society for Heating Refrigeration and Air conditioning Engineers (ASHRAE) defined operating range that different types of data centers can operate. The range specifies how climatic condition can affect data center cooling. To exploit advantage of data center free cooling temperature characteristics of data center expected location should be monitored. For a case of HLIT climatic condition of each zone is as indicated in Table 1.

Table 1 Tanzania average regional Temperature. [Source: TMA1]

\begin{tabular}{|c|c|c|c|}
\hline & Zone Name & AVR Min Temp & AVR Max Temp \\
\hline 1 & Western & 16 & 25 \\
\hline 2 & Eastern & 25 & 33 \\
\hline 3 & Southern & 17 & 27 \\
\hline 4 & Northern & 20 & 27 \\
\hline 5 & Central & 18 & 27 \\
\hline
\end{tabular}

In order to have eco-aware data centers; the use of the nature of climatic conditions in identifying a possible cooling framework should be in accordance to the $[16,17]$ guidelines. The free cooling strategy has been used to lower Power Unity Effectiveness (PUE) of data centers. Free cooling involves the ability to use local ambient conditions to remove heat from inside a data center $[18,17]$. To relate cooling and PUE we have,

$$
P U E=\frac{T F E}{I T E},
$$

where TFE is total facility energy, and ITE is IT energy. TFE includes power that is used by data center supporting infrastructure such as cooling system and Uninterrupted Power Supply (UPS). Use of air conditioners and electrical fans raises TFE. Free cooling lowers PUE by minimizing TFE through the use of climatic ambient conditions.

\footnotetext{
${ }^{1}$ Tanzania Meteorological Agency (2014)
}

There are two forms of free cooling techniques, air-side and water-side cooling. Air-side cooling uses air economizer to bring the coldness of outside air into the data center to cool servers below the specification indicated in ASHRAE'S thermal guidelines (The 42u.com, 2014). Water-side economizers use cold air to cool an exterior water tower. Water side economizer gives good result for the climatic condition where temperature is below 12.78 degree centigrade for 3000 hours or more [19]. In HLIT environment, the average temperature range is above specified requirement, thus we won't consider water economizer in our work. The next part analyzes the use of air-side economizer as a cooling strategy for private data centers in HLIT clouds.

Air-side economizer pumps fresh air into a data center and hot air is exhausted. A system that can deliver $3 \mathrm{~m} 3$ of air can consume $1.5 \mathrm{~kW}$, and save up to $30 \mathrm{~kW}$ [20]. For the climatic conditions described in [21], relying on air economizer in $91 \%$ of the year can save approximately $67 \%$ of the total power used annually for cooling compared with a traditional data center cooling approaches. Temperature characteristics of possible data center location as seen in Table 1 prove the feasibility of using air-side economizers. The minimum temperature in all zones is within ASHRAE recommended range. Similarly, the maximum temperature of zones except eastern is below maximum ASHRAE recommended range. For full use of economizer, one host may reallocate its workload to another host in a location where the use of an air-side economizer is feasible. During the night, temperature is considerably low, (minimum zone temperature in Table 1) therefore, air-side economizers can be used in all zones.

\subsection{Data Center Layout for Free Cooling}

One major data center design principle is efficient air flow management within a data center that can be achieved by optimal positioning of data center equipment [22]. Air flow management entails all the design and configuration details that go into minimizing or eliminating a mixture between the cooling air supplied to equipment and the hot air rejected from the equipment. To achieve economical cooling circulation of air, the cooling system should be able to remove hot exhaust air before exhausting, and the heat it carries is mixed with cool fresh air. To make sure that drawing and exhaust airs are not mixed, data center equipment is laid out in rows of racks with alternating cold (rack air intake side) and hot (rack air heat exhaust side) aisles between them; as shown in Fig 2. 


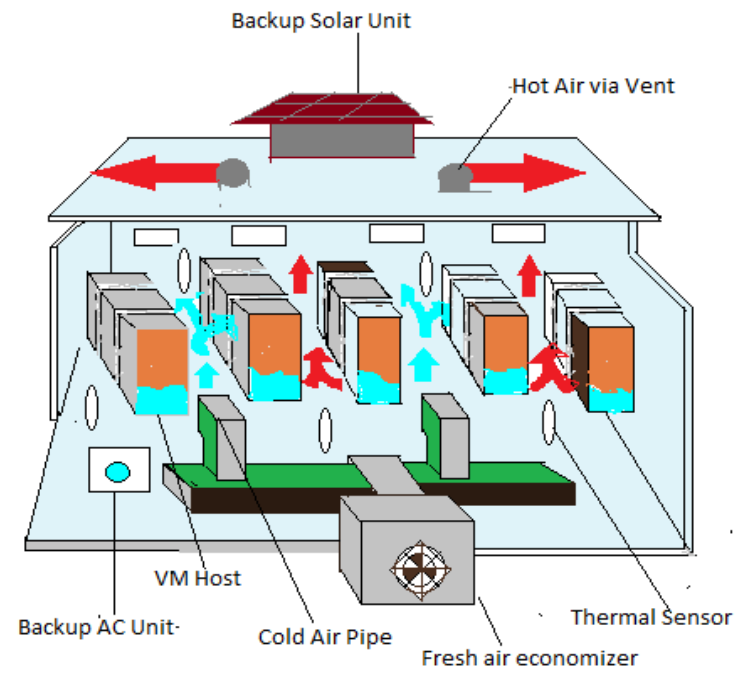

Fig 2 Efficient cooling model for data center

Air-side economizer draws outside air and distributes between and above the racks. Exhausted hot air is pushed out of the racks via a vent. To ensure service availability safety of data center during climatic variations, backup AC unit can be incorporated. AC should be powered using renewable power sources to avoid addition of carbon foot print during its operation. Distance between server and racks, racks and racks, server and server should be enough for smooth maintenance and server configuration. Air-side economizer distribution unity can be overhead or underfloor depending on the type of the rack used to allow effective cooling.

\subsection{Network Coverage}

VM migration requires a high-bandwidth connection from user to destination data centers [8]. In order to maximize data center efficiency, a cloud user request should be processed in the data center that have optimum resource cost while ensuring minimum migration overhead. Let $\alpha$ describe the cost associated with single VM migration across a network, then:

$\alpha=<V, N s, N d, B, D, T>$,

where, $\mathrm{V}$ is the VM to be migrated to, Ns is the source location, $\mathrm{Nd}$ is the destination location, $\mathrm{B}$ is the required connection bandwidth, $\mathrm{D}$ is the connection holding duration, and $\mathrm{T}$ is the time when the migration shall start. Thus, the total data volume to be transferred if the request is satisfied is given by $\mathrm{B} \times \mathrm{D}$ [8].

Any placement of a VM to a host should minimize migration cost while taking care of performance and quality of services (QoS). For the case of HLIT, it was found that cloud bandwidth requirements in all zones can be achieved based on accessibility of the NICTBB. In addition, the nature of power sources that are available supports the use of renewable energy sources to power cloud data centers. Having network infrastructure support, enriches zoning data center placement strategy that makes efficient use of available resources by allowing flexibility in consolidation of VMs from one zone to another when cloud services are not meeting desired requirements.

\subsection{Quality of Service}

While optimal location of data center, network coverage and nature of power source are important data center building blocks, quality of service during data center operation should be achieved. Virtualization of computing services should not impact performance that a user experiences on using non virtualized services. The percentage by which virtual machine consolidation is violating the SLA should be considered while minimizing cloud data center energy usage and carbon emission. Usually, QoS can be degraded by the following factors: (1) the fraction of time during which active hosts have experienced CPU utilization of $100 \%$, i.e., Overload Time Fraction (OTF); and (2) the overall performance degradation by VMs due to migration, i.e., Performance Degradation due to Migrations (PDM) [23]. OTF and PDM metrics should be optimized by having an efficient resource allocation strategy.

\subsection{HLIT Cloud Software (OS, management tool, hypervisor)}

When deciding the best possible design for building HLIT hybrid cloud, we came across a wide range of cloud management and monitoring tools, and operating systems for which to install these components. Due to the heterogeneity of cloud computing, it is important to choose a suitable tools platform to manage virtual machines and hosts. In this study, various tools were identified. Their analysis led to the selection of those in favor of our design environment and objective.

\subsubsection{Operating System}

One of the most important ways to support the underlying complexity of well-managed cloud computing resources is through the operating system (OS) [24]. Operating system such as Debian, Openness, Ubuntu Server, Linux and others are created to support these requirements so that cloud and application services should not recreate underlined technologies tailored to each deployment. The best OS support balanced workloads and can scale in a secure manner.

Debian is a free and open source operating system which includes GNU operating system tools and a Linux kernel. It is compatible with Xen and has a GUI integrated with it. Openness is also a free and open source operating system made up of a series of software packages. It includes GNU operating system tools and a Linux kernel. It is compatible with Xen and includes both a default Graphical User Interface (GUI) and a command line interface option. Ubuntu Server Edition also runs on VMware ESX Server, Oracle's VirtualBox and VM, Citrix Systems XenServer hypervisors, Microsoft Hyper-V as well as Kernel-based Virtual Machine [25]. Ubuntu server supports a major two architectures; Intel x86 and AMD64. The server edition uses a screen mode character-based interface for the installation instead of a graphical installation process. It consists of open core Eucalyptus, libvirt, and KVM or Xen virtualization technology.

Reilly and Cearra, (2012) investigated different operating systems in order to test which one is suitable for hybrid cloud infrastructure. From the experiment, Ubuntu Server was found to be the most efficient operating system under a hybrid cloud environment. As a point to remember, Ubuntu server is a FOSS. It is furthermore compatible with other cloud software's such as Xen, Open Nebula, CloudStack and others. This makes a strong point to propose Ubuntu server to be an operating system to run on servers for hybrid cloud computing for HLIT.

\subsubsection{Cloud management tool (CloudStack)}

Building a data center in a distributed environment raises the need of software which can manage the complexities and the heterogeneity of cloud workload in a mixed demand environment. 
The candidate that can fit in that environment is an Open-source software called CloudStack. CloudStack is a software platform that pools computing resources to build public, private and hybrid IaaS clouds [26]. CloudStack gives a cloud developer the ability to extend the cloud resources allocation policy through the use of pluggable allocation architecture that allows the creation of new types of allocation policy. It supports zoning architecture which is the desirable model for HLIT. It divides cloud infrastructure into Zone, Pod, Cluster and final host. Its nested architecture gives high flexibility in managing and configuring cloud resources. In addition, CloudStack is built with a number of VM monitors, and network configuration features that are flexible to multiple design environments.

\subsection{Data center distribution architecture}

Fig 3 shows the proposed data center distribution in different zones of the country. Each data center placement is influenced by accessibility to NICTBB and availability of renewable power resources and ambient climatic conditions that allow free cooling.

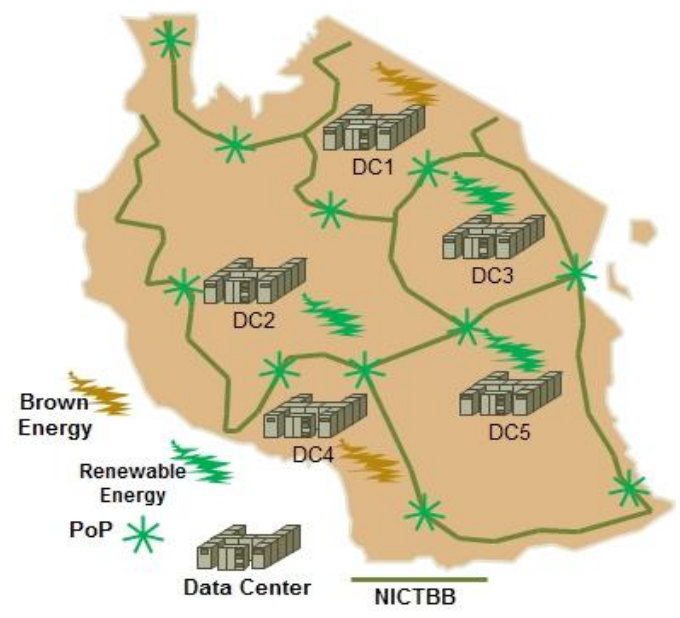

Fig 3 HLIT Cloud Computing Data centers distribution

\section{Related works}

Several cloud academic computing frameworks have been proposed [13, 27, 1]. With similar concerns of establishing efficient computing systems for academic services; Saidhbi (2012) proposed alternative solutions to solve the current IT utilization, and limitations in Ethiopian Higher Education Institutions. The author proposed hybrid cloud framework to enhance academic requirements. The proposed framework combines multiple services from different cloud service providers (CSPs) to serve students and other users from different universities to enhance the teaching, learning and service delivery. OpenNebula is the proposed cloud management tool; on top of OpenNebula, Aneka middleware layer is added to manage PaaS. The framework is designed to utilize power aware techniques that are in OpenNebula. Suryawanshi and Narkehde, (2012) proposed the cloud framework for higher technical education. The framework includes three deployment models (i.e. private, public and hybrid). The framework is structured into four layers SaaS, PaaS, IaaS and hardware. SaaS layer is divided into four components which are faculty, student, staff and research. The suggested framework does not address today's data center power consumption problems. However, Beloglazov et al, (2012) and Esfandiarpoor et al (2013) recommended power aware data center heuristics that reduce carbon dioxide and power consumption. In the work, performance and QoS of a data center were analyzed. Virtual machine consolidation heuristics were developed. The heuristics lower power consumption of host by shutting down underutilized and idle servers.

Choosing cloud solution according to its usage environment is very important on selecting cloud building blocks. Singh and Hemalatha, (2012) suggested academic cloud that suits storage infrastructure, development platform, and software delivering environment. The proposed framework separates user data on cloud by providing user with a password secured virtual storage. The framework was enriched with high performance features in order to outperform thin client computing system being used in academic environment. Open source solution has been used by Pantić and Babar, (2012) to develop private cloud infrastructure that suits organizational environment. Building blocks of the proposed private data center were made up with Ubuntu Enterprise Cloud (UEC), which is a combination of Ubuntu Server Edition with Eucalyptus. Bundling blocks make UEC easier to install and configure the cloud. On the other hand, it uses the same API's as Amazon making hybrid cloud solution easy to implement. The author suggested other building blocks that included specifications of efficient hardware, UEC building and configuration, network requirements that are suitable for private and small organization.

The similarities and differences of the identified works with our presented eco-efficient building blocks are that; both work addressed TC challenges. Likewise, both work focused on cost effective cloud solution where free and open source cloud infrastructure has been preferred. The difference is that; our building blocks encompassed use of thermal VM consolidation and Green aware power sources in building private data center that suits HLIT.

\section{Conclusion and Future Work}

In this chapter, we have leveraged technology, environment and infrastructure to form eco-efficient and cost effective cloud computing building blocks for HLIT. The building blocks examine the basic consideration that are important in ensuring: 1) challenges that are in HLIT traditional computing are addressed in academic cloud framework; 2) Challenges that have already been presented in cloud computing framework including huge power consumption and high carbon emission are addressed in academic eco-aware framework; 3) academic framework takes advantage of environment conditions in minimizing power utilization. To ensure that stated purposes are met we identified six environments, Technology and Infrastructure building blocks. We have analyzed the nature of power to be used to power cloud data center that consider green power availability and its performance implications to cloud operations. We have taken a look on cloud deployment model and data center location that consider power sources that are available in each identified region. Next we have considered ambient climatic conditions and data center cooling. In our approach, we have explored best cooling practices that can be exploited to lower non IT facility power consumption in data centers. Besides, we have observed how ambient temperature affects free cooling. We came up with model that can enhance free cooling practice and that is in accordance with ASHRAE (2008) guidelines.

Our focus in this work was on eco-efficient but we cannot have efficient cloud without considering service delivery 
infrastructure. Therefore, we have considered blocks that ensure quality of service and cloud efficient measurement. Cloud computing services are provisioned over the internet, high bandwidth connectivity as important criteria to locate a data center. We examined a network connection that can support cloud infrastructure. The presence of NICTBB in many regions in Tanzania makes data center zoning architecture across multiple geographic locations feasible. The quality of service and tradeoffs caused by virtualization has been described; quality of service consideration gives consciousness on performance and SLA violation when consolidating virtual machine.

Lastly, we have considered software necessary for building cloud computing for HLIT. Free-Open source software has been trusted in our work due to its flexibility and compatibility across multiple hardware settings. OpenStack gives many programming extensible features that can be used to create green resources cloud allocator that is suitable for a proposed zoning architecture. Furthermore, it allows cloud developers to create and manage cloud services that are distributed in a wide geographic area.

\section{References}

[1] A. Singh and M. Hemalatha, "Cloud Computing for Academic Environment," International Journal of Information and Communication Technology Research, vol. 2, no. 2, pp. 97-101, 2012.

[2] M. Mircea and A. Andreescu, "Using Cloud Computing in Higher Education: A Strategy to Improve Agility," vol. 2011, 2011.

[3] M. Mircea, "SOA,BPM and Cloud Computing : Connected for Innovation in Higher Education," in IEEE International conference on Education and Management Technology ICEMT, 2010.

[4] R. Mero and J. Mwangoka, "Road Map towards Ecoefficient Cloud Computing Adoption in Higher Learning Institutions in Tanzania," Pan African International Conference on Information Science, Computing and Telecommunications (PACT 2014) Arusha, 2014.

[5] J. Pagare and A. Koli, "Energy-Efficient Cloud Computing: A Vision, Introduction, and Open Challenges," International Journal of Computer Science and Network(IJCSN), vol. 2, no. 2, pp. 96-102, 2013.

[6] M. Kaplan, W. Forrest and N. Kindler, "Revolutionizing Data Center Energy Efficiency," McKinsey \& Company, 2008.

[7] Z. Pantić and M. Babar, "Guidelines for Building a Private Cloud Infrastructure," Copenhagen, Denmark, 2012.

[8] U. Mandal, M. Habib, S. Zhang, M. Tornatore and B. Mukherjee, "Greening the Cloud Using RenewableEnergy-Aware Service Migration," Davis, 2013.

[9] Worldenergyoutlook, "World energy outlook," 2008.

[10] IndexMund, "World Energy Report," Index Mund inc, 2010.
In this chapter, we have identified and discussed HLIT ecoefficient cloud building blocks and a possible cloud deployment model, i.e. Hybrid cloud has been proposed. Zoning architecture is suggested data center location strategy, where private data center will be built in a region that meets eco-efficient requirements such as availability of green power sources and ambient temperature that can be used for free cooling. In the anticipated architecture, seasonal climatic condition variation presents challenges and opportunities in free cooling strategy. Moreover, unpredictable workloads in a data center present a cloud service provisioning challenge. Thus, there has to be a VM consolidation mechanism that considers the challenges. Our future work, therefore, is to create green aware consolidation heuristic that fits in the described environment. The heuristic will consider green aware power sourced data center, and free cooling in virtual machine consolidation to reduce data center power consumption and carbon footprints. The heuristic will also optimize power consumption and carbon emission in the cloud data center.

[11] C. Wolfram, O. Shelef and P. Gertler, "How Will Energy Demand Develop in the Developing World?," 2012.

[12] M. Albaijat, "Optimal Wind Energy Intergaration in Large-sxale Electric Grids, Ph.D dissertation," UC David, chicago, 2013.

[13] S. Saidhbi, "A Cloud Computing Framework for Ethiopian Higher Education Institutions," IOSR Journal of Computer Engineering (IOSRJCE), vol. 6, no. 6, pp. 1-9, 2012.

[14] D. Bouley, "Estimating a Data Center's Electrical Carbon Footprint. Schneider Electric - Data Center Science Center," White Paper, no. 66, 2012.

[15] TheGreenGrid, "Data center efficiency and It equipment reliability at Wider operating Temperature and humidity Ranges," Green Greed Whitepaper, no. 50, 2012.

[16] ASHRAE, "Second edition of the Thermal Guidelines for Data Processing Environments.," ASHRAE , newyork, 2008.

[17] ASHRAE, "2011 Thermal Guidelines for Data Processing Environments â€" Expanded Data Center Classes and Usage Guidance. White paper," ASHRAE, Newyork, 2011.

[18] HP, "Applying 2011 ASHRAE data center guidelines to HP ProLiant-based facilities," White paper, 2012.

[19] Energystar, "energystar.gov," 2014. [Online]. Available: http://www.energystar.gov/index.cfm?c=power_mgt.data center_efficiency_economizer_waterside. [Accessed 4 April 2014].

[20] Z. Potts, "Free Cooling Technologies in Data Centre Applications," 2011.

[21] D. Atwood and J. Miner, "Reducing Data Center Cost with an Air Economizer.," Intel Information Technology., 2008.

[22] Rumsey-Engineers, High Performance DataCenters a Design Guidelines Sourcebook, Pacific Gas and Electric Company, 2006. 
[23] A. Beloglazov, J. Abawajy and R. Buyya, "Energy-Aware Resource Allocation Heuristics for Efficient Management of DataCenters for Cloud Computing," Future Generation Computer Systems (FGCS), vol. 28, no. 5, pp. 755-768., 2012.

[24] J. Hurwitz, "The Role of the Operating System in Cloud Environments," White paper, 2011.

[25] M. Reilly and M. Cearra, "Opennebula and The Xen Hypervisor," 2012.

[26] Cloudstack, "Cloudstack.apache.org," 2014. [Online]. Available: $\quad$ http://Cloudstack.apache.org/docs/enUS/Apache_CloudStack/4.0.0-incubating/htmlsingle/Admin_Guide. [Accessed 4 April 2014].

[27] K. Suryawanshi and S. Narkehde, "A Study of Green ICT and Cloud Computing Implementation at Higher Technical Education Institution," IJARCET, vol. 1, no. 8, pp. 377-382, 2012.

[28] J. Ward and A. Barker, "A Cloud Computing Survey: Developments and Future Trends in Infrastructure as a Service Computing," arXiv preprint arXiv:1306.1394, 2013.

[29] W. Voorsluys, J. Broberg, S. Venugopal and R. Buyya, "Cost of virtual machine live migration in Clouds: A performance evaluation," in in Proceedings of the 1st International Conference on Cloud Computing (CloudCom), 2009.

[30] VMware, "A Guide to Large-scale Enterprise VMware View 3 and VMware View 4 Deployments," VMware, Inc, 2010.

[31] E. Uyigue, M. Agho, A. Edevbaro, O. Godfrey, O. Uyigue and O. Okungbowa, "Energy Efficiency Survey in Nigeria. Community Research and Development Centre," Community Research and Development Centre, Edo State, Nigeria, 2009.

[32] SPEC, "Standard Performance Evaluation Corporation," 2014 [Online]. Available: http://www.spec.org/power_ssj2008/. [Accessed 3 jun 2014].

[33] L. Shang, L.-S. Peh and N. K. Jha, "Dynamic voltage scaling with links for power optimization of interconnection networks," in In: Proceedings of the 9th international symposium on high performance, 2003.

[34] T. Samir, "A Roadmap for Transitioning an Information Assurance Program and Others to," International Journal of Information and Communication Technology Research, vol. 1, no. 3, pp. 128-138, 2011.

[35] P. Ranganathan, P. Leech, D. Irwin and J. Chase, "Ensemble-level power management for dense blade servers," in in Proceedings of the 33rd International Symposium on Computer Architecture (ISCA), 2006.

[36] C. D. Patel, C. Bash and A. Beitelmal, "Smart cooling of data centers," Google Patents, 2003.

[37] J. Pagare and A. Koli, "Energy-Efficient Cloud Computing: A Vision, Introduction, and Open Challenges," International Journal of Computer Science and Network, vol. 2, no. 2, pp. 96-102, 2013.
[38] M. Myo and T. Thein, "Efficient Resource Allocation for Green Cloud Data Center," in 3rd International Conference on Computational Techniques and Artificial Intelligence(ICCT), 2014.

[39] S. Mrdalj, "Would cloud computing revolutionize teaching business intelligence courses," Issues in Informing Science and Information Technology, vol. 8, 2011.

[40] R. Mero and J. Mwangoka, "Building Block for Ecoefficient Cloud Computing Framework for Higher Learning Institutions in Tanzania," Unpublished, 2014.

[41] P. Mell and T. Grance, "The NIST Definition of cloud computing," 2011.

[42] C.-C. Lin, P. Liu and W. J-J, "Energy-Aware Virtual Machine Dynamic Provision and Scheduling for Cloud Computing," in 2011 IEEE 4th International Conference on Cloud Computing, 2011.

[43] D. Kliazovich, P. Bouvry and S. Khan, "DENS: Data Center Energy-Efficient Network-Aware Scheduling," in IEEE/ACM International Conference on Green Computing and Communications (GreenCom) \& International Conference on Cyber, Physical and Social Computing (CPSCom), 2010.

[44] P. Kish, "PUE Standards for Energy Efficient Data Centers on the Horizon," 2013.

[45] A. Imenez-Castellanos, G. de la Calle, R. Alonso-Calvo, R. Hussein and V. Maojo, "Accessing advanced computational resources in Africa through Cloud Computing," Computer-Based Medical Systems (CBMS), pp. 1-4; 20-22, 2012.

[46] J. Hamilton, "ooperative expendable micro-slice servers(CEMS): low cost, low power servers for Internetscale services," in CIDR Conference, 2009.

[47] X. Fan, W. D. Weber and L. A. Barroso, "Power provisioning for a warehouse-sized computer," in in Proceedings of the 34th Annual International Symposium on Computer Architecture (ISCA), 2007.

[48] S. Esfandiarpoor, A. Pahlavan and M. Goudarzi, "Virtual Machine Consolidation for Datacenter Energy Improvement," 2013.

[49] eia, "International Energy Outlook 2013," U.S departiment of energy 1000 independent Av, SW, Washinton DC, 2013.

[50] Cisco, "Cloud 101: Developing a Cloud-Computing Strategy for Higher Education white paper Cloud 101," 2012.

[51] M. Cardosa, M. Korupolu and A. Singh, "Shares and utilities based power consolidation in virtualized server environments," in: Proceedings of the 11thIFIP/IEEE Integrated Network Management, 2009.

[52] M. Bluckburn, "Five ways to reduce data center server power consumption. The Green Grid," 2008.

[53] A. Beloglazov, R. Buyya, L. Choon and Z. Albert, "Taxonomy and Survey of Energy-Efficient Data Centers and Cloud Computing Systems," Future Generation Computer Systems (FGCS), vol. 1, no. 2, pp. 1-52, 2010. 
International Journal of Computer Applications Technology and Research

Volume 7-Issue 04, 163-170, 2018, ISSN:-2319-8656

[54] Australia, "Australian Government Data Centre Strategy 2010-2025. Better Practice Guide: Data Centre Cooling.," Australia Gov, Australia, 2013.

[55] 42u.com, "www.42u.com," 2014. [Online]. Available: http://www.42u.com/cooling/economizers/economizers.h tm. [Accessed 04 April 2014].

[56] R. Suryawanshi, P. Choudhary and Naidu, "An Analysis and Implementation of Cloud Computing at Higher Technical Education," International Journal of Computer
Applications and Business Intelligence (IJCABI), vol. 2, pp. 13-19, 2012.

[57] N. Ajiths and M. Hemalatha, "Cloud Computing for Academic Environment," International Journal of Information and Communication Technology Research, vol. 2, no. 2, pp. 97-101, 2012. 